\title{
Exact black holes in quadratic gravity with any cosmological constant
}

\author{
R. Švarc, J. Podolsky* \\ Institute of Theoretical Physics, Charles University, Prague, \\ Faculty of Mathematics and Physics, V Holešovičkách 2, 18000 Prague 8, Czech Republic \\ V. Pravda, A. Pravdovê门 \\ Institute of Mathematics of the Czech Academy of Sciences, Žitná 25, 11567 Prague 1, Czech Republic
}

(Dated: November 14, 2018)

\begin{abstract}
We present a new explicit class of black holes in general quadratic gravity with a cosmological constant. These spherically symmetric Schwarzschild-Bach-(anti-)de Sitter geometries (Schwa-Bach(A)dS), derived under the assumption of constant scalar curvature, form a three-parameter family determined by the black-hole horizon position, the value of Bach invariant on the horizon, and the cosmological constant. Using a conformal to Kundt metric ansatz, the fourth-order field equations simplify to a compact autonomous system. Its solutions are found as power series, enabling us to directly set the Bach parameter and/or cosmological constant equal to zero. To interpret these spacetimes, we analyse the metric functions. In particular, we demonstrate that for a certain range of positive cosmological constant there are both black-hole and cosmological horizons, with a static region between them. The tidal effects on free test particles and basic thermodynamic quantities are also determined.
\end{abstract}

PACS numbers: 04.20.Jb, 04.50.-h, 04.50.Kd, 04.70.Bw, 04.70.Dy

\section{INTRODUCTION}

Black holes, regions with very strong gravity from which not even light can escape, are one of the most fascinating theoretical predictions of Einstein's general relativity [1. The first exact solution to this theory was almost immediately found by Schwarzschild [2, describing a static spherically symmetric spacetime. However, it took several decades to fully understand its black-hole nature. This initiated the 'golden age' of black hole studies, epitomized by the discovery of astrophysically more relevant Kerr rotating solution [3. Studies of various aspects of these 'collapsed objects', such as influence on matter and fields, the no-hair conjecture, thermodynamic properties, or quantum evaporation, followed soon. Moreover, a great observational effort brought the direct evidence of their existence in our universe when Cygnus X-1 source was identified as a black hole. Also, now it seems that supermassive black holes reside in nuclei of almost all galaxies. Mergers of black-hole binaries have been recently detected as the first gravitational wave signals.

Another remarkable interplay between Einstein's theory and observed astronomical phenomena is a concept of the cosmological constant. The famous $\Lambda$-term was introduced by Einstein into his field equations to allow a static cosmological model [4. However, it was soon demonstrated by de Sitter [5] that the cosmological constant causes even an empty space to expand exponentially fast 6. Nowadays, this is employed for a phenomenological description of the observed accelerated expansion of our universe caused by 'dark energy'. The de Sitter solution

* robert.svarc@mff.cuni.cz, podolsky@mbox.troja.mff.cuni.cz

$\dagger$ pravda@math.cas.cz pravdova@math.cas.cz also captures main features of the inflationary epoch in the very early universe.

Despite all the great successes of Einstein's gravity theory, it also has its limits, in particular, impossibility to quantize it in the same way as other fundamental interactions, and perhaps some open cosmological issues. Various extensions of general relativity have thus been considered, see 7-10 for reviews. In these modified theories, the black hole solutions play a prominent role, providing natural test beds for their comparison [11 14].

Assuming a constant scalar curvature, we derive a new class of static spherically symmetric black hole solutions with a cosmological constant $\Lambda$ in quadratic gravity [15], which includes the Einstein-Weyl theory [16, 17. It generalizes 18 to include higher-order gravity corrections, and 14, 19] to admit any $\Lambda$. In contrast with the black holes of [14, 19], the second (cosmological) horizon may appear due to $\Lambda>0$. On large scales, the higher-order corrections considerably affect the asymptotic behaviour of the geometry, which, even in the case of $\Lambda=0$, is not asymptotically flat (except for finely tuned parameters). This additional freedom thus opens completely new and more involved possibilities. Moreover, both the cosmological constant and higher-order corrections are of key importance in quantum gravity models, e.g., 20].

Within this setting, the vacuum action of quadratic gravity contains $\Lambda$, the Ricci scalar $R$, and a contraction of the Weyl tensor $C_{a b c d}$, namely

$$
S=\int \mathrm{d}^{4} x \sqrt{-g}\left(\gamma(R-2 \Lambda)+\beta R^{2}-\alpha C_{a b c d} C^{a b c d}\right),
$$

where $\alpha, \beta, \gamma=G^{-1}$ are constants. The corresponding 
field equations read

$$
\begin{aligned}
& \gamma\left(R_{a b}-\frac{1}{2} R g_{a b}+\Lambda g_{a b}\right)-4 \alpha B_{a b} \\
& \quad+2 \beta\left(R_{a b}-\frac{1}{4} R g_{a b}+g_{a b} \square-\nabla_{b} \nabla_{a}\right) R=0,
\end{aligned}
$$

where $B_{a b} \equiv\left(\nabla^{c} \nabla^{d}+\frac{1}{2} R^{c d}\right) C_{a c b d}$ is the traceless, symmetric and conserved Bach tensor. Assuming $R=$ const., the last term in 1.2 simplifies and the trace of the field equations implies $R=4 \Lambda$, so that they become

$$
R_{a b}-\Lambda g_{a b}=4 k B_{a b}, \quad \text { with } \quad k \equiv \frac{\alpha}{\gamma+8 \beta \Lambda},
$$

see [21]. For $k=0$ vacuum Einstein's equations with a cosmological constant are obtained. For $\beta=0$ we get Einstein-Weyl gravity. For $\gamma+8 \beta \Lambda=0$ the conformal Weyl theory is restored, in which the rotational curves of galaxies were studied [23] within the spherically symmetric setting. Our solution, as an unifying model, may enable the analysis of relations between these theories in such astrophysical situations.

\section{THE GEOMETRY}

A spherically symmetric metric is usually written as

$$
\mathrm{d} s^{2}=-h(\bar{r}) \mathrm{d} t^{2}+\frac{\mathrm{d} \bar{r}^{2}}{f(\bar{r})}+\bar{r}^{2}\left(\mathrm{~d} \theta^{2}+\sin ^{2} \theta \mathrm{d} \phi^{2}\right) .
$$

However, in [19, 22 it was shown that for investigation of such geometries in quadratic gravity, an alternative form is more convenient,

$$
\mathrm{d} s^{2}=\Omega^{2}(r)\left[\mathrm{d} \theta^{2}+\sin ^{2} \theta \mathrm{d} \phi^{2}-2 \mathrm{~d} u \mathrm{~d} r+\mathcal{H}(r) \mathrm{d} u^{2}\right] .
$$

This is related to 2.1 via

$$
\bar{r}=\Omega(r), \quad t=u-\int \mathcal{H}(r)^{-1} \mathrm{~d} r
$$

and the metric functions $\Omega, \mathcal{H}$ give $f, h$ using

$$
h(\bar{r})=-\Omega^{2} \mathcal{H}, \quad f(\bar{r})=-\left(\frac{\Omega^{\prime}}{\Omega}\right)^{2} \mathcal{H}
$$

(prime denotes the derivative with respect to $r$ ). The new metric 2.2 is conformal to a simple direct-product Kundt 'seed', $\mathrm{d} s^{2}=\Omega^{2} \mathrm{~d} s_{\text {Kundt }}^{2}$, which is of the algebraic type D, see [21, 24, 25].

In the metric 2.2, the Killing horizons corresponding to $\partial_{u}=\partial_{t}$ are located at specific radii $r_{h}$ satisfying

$$
\left.\mathcal{H}\right|_{r=r_{h}}=0
$$

Of course, via (2.4) this gives $h\left(\bar{r}_{h}\right)=0=f\left(\bar{r}_{h}\right)$. There is a time-scaling freedom $t \rightarrow \sigma^{-1} t$ of the metric 2.1 implying $h \rightarrow \sigma^{2} h$, which can be used, e.g., to adjust appropriate value of $h$ at a chosen radius.
To uniquely characterize the geometries 2.2, we need the Weyl and Bach scalar curvature invariants,

$$
\begin{aligned}
C_{a b c d} C^{a b c d} & =\frac{1}{3} \Omega^{-4}\left(\mathcal{H}^{\prime \prime}+2\right)^{2}, \\
B_{a b} B^{a b} & =\frac{1}{72} \Omega^{-8}\left[\left(\mathcal{B}_{1}\right)^{2}+2\left(\mathcal{B}_{1}+\mathcal{B}_{2}\right)^{2}\right],
\end{aligned}
$$

where two independent Bach components are

$$
\mathcal{B}_{1} \equiv \mathcal{H} \mathcal{H}^{\prime \prime \prime \prime}, \quad \mathcal{B}_{2} \equiv \mathcal{H}^{\prime} \mathcal{H}^{\prime \prime \prime}-\frac{1}{2} \mathcal{H}^{\prime \prime 2}+2 .
$$

Interestingly, $B_{a b}=0 \Leftrightarrow B_{a b} B^{a b}=0$. Thus we distinguish two geometrically different types of solutions in quadratic gravity defined by $B_{a b}=0$ and $B_{a b} \neq 0$, respectively.

\section{THE FIELD EQUATIONS}

Under conformal transformations, the Bach tensor simply scales as $B_{a b}=\Omega^{-2} B_{a b}^{\text {Kundt }}$ and since higher-order corrections in 1.3 are represented by the Bach tensor, using the metric (2.2) leads to a remarkable simplification of the field equations. Explicit evaluation of the field equations 1.3 for 2.2 , using the Bianchi identities, yields two simple ODEs for the metric functions $\Omega(r)$ and $\mathcal{H}(r)$, namely

$$
\begin{aligned}
\Omega \Omega^{\prime \prime}-2 \Omega^{\prime 2} & =\frac{1}{3} k \mathcal{B}_{1} \mathcal{H}^{-1}, \\
\Omega \Omega^{\prime} \mathcal{H}^{\prime}+3 \Omega^{\prime 2} \mathcal{H}+\Omega^{2}-\Lambda \Omega^{4} & =\frac{1}{3} k \mathcal{B}_{2},
\end{aligned}
$$

see [26] for more details. It is also convenient to express the trace of 1.3 , namely $R=4 \Lambda$,

$$
\mathcal{H} \Omega^{\prime \prime}+\mathcal{H}^{\prime} \Omega^{\prime}+\frac{1}{6}\left(\mathcal{H}^{\prime \prime}+2\right) \Omega=\frac{2}{3} \Lambda \Omega^{3}
$$

In fact, it is the derivative of 3.2 minus $\mathcal{H}^{\prime}$ times 3.1. The crucial point for further investigations is that Eqs. 3.1 (3.2) do not explicitly depend on $r$. Solutions to such an autonomous system can thus be found as power series in $r$ expanded around any point $r_{0}$

$$
\Omega(r)=\Delta^{n} \sum_{i=0}^{\infty} a_{i} \Delta^{i}, \quad \mathcal{H}(r)=\Delta^{p} \sum_{i=0}^{\infty} c_{i} \Delta^{i},
$$

where $\Delta \equiv r-r_{0}, n, p \in \mathbb{R}$, and $a_{0}, c_{0} \neq 0$.

\section{A. Vanishing Bach tensor}

For $\mathcal{B}_{1}=0=\mathcal{B}_{2}$, we deal with Einstein's theory, and Eqs. 3.1, 3.2 can be directly integrated. Using the gauge freedom $r \rightarrow \lambda r+\nu, u \rightarrow \lambda^{-1} u$ of the metric 2.2, this immediately implies

$$
\Omega(r)=\bar{r}=-\frac{1}{r}, \quad \mathcal{H}(r)=\frac{\Lambda}{3}-r^{2}-2 m r^{3},
$$

where the mass parameter $m$ is fixed by (2.5), see 4.3 . These functions represent the Schwarzschild-(anti-)de 
Sitter spacetime [18, 24, 25] which, expressed in the form (2.1) using 2.4, reads $f=h=1-2 m \bar{r}^{-1}-\frac{1}{3} \Lambda \bar{r}^{2}$.

It is well known 25 that for $0<9 \Lambda m^{2}<1$ there are two horizons determined by (2.5), namely the blackhole event horizon at $r_{h}$ and the cosmological horizon at $r_{c}>r_{h}$ (they degenerate to $\bar{r}_{h}=\bar{r}_{c}=3 m=1 / \sqrt{\Lambda}$ when $9 \Lambda m^{2}=1 ; \Lambda<0$ admits only the black hole horizon).

\section{B. Non-vanishing Bach tensor}

In a generic case $\left(\mathcal{B}_{1}, \mathcal{B}_{2} \neq 0\right)$, the system $(3.1),(3.2)$ becomes non-trivially coupled but its solutions can be found in the form (3.4). Substituting these series into the field equations, we obtain polynomial expressions where the dominant (lowest) powers of $\Delta$ immediately put specific restrictions on the parameters $[n, p]$ and the possible value of $\Lambda$, see Tab. II and [26]. In the next section, we will discuss the most interesting case $[0,1]$ corresponding to a single root $r_{0}$ of 2.5 .

TABLE I. The only admitted parameters $[n, p]$ in 3.4 , and the cosmological constant $\Lambda$, restricted by dominant powers of $\Delta$ in the field equations $3.1,3.2$, and the trace 3.3 . Note that in the last column, $n \neq-1,-1 / 2$.

\begin{tabular}{c|cccccccc}
$n$ & 0 & 0 & 1 & -1 & -1 & 0 & 0 & $<0$ \\
\hline$p$ & 1 & 0 & 0 & 2 & 0 & 2 & $\geq 2$ & $2 n+2$ \\
\hline$\Lambda$ & any & any & any & 0 & $\neq 0$ & $\neq 0$ & $\frac{3}{8 k}$ & $\frac{11 n^{2}+6 n+1}{1-4 n^{2}} \frac{3}{8 k}$
\end{tabular}

\section{EXPLICIT BLACK HOLES}

In the case $n=0, p=1$, the root of $\mathcal{H}$ representing the non-degenerate Killing horizon (2.5) is explicitly given by $r_{0} \equiv r_{h}$. The field equations (3.1), (3.2), with (3.3), then restrict the coefficients in the expansions (3.4) as

$$
\begin{aligned}
a_{1}= & \frac{1}{3 c_{0}}\left[2 \Lambda a_{0}^{3}-a_{0}\left(1+c_{1}\right)\right], \\
c_{2}= & \frac{1}{6 k c_{0}}\left[a_{0}^{2}\left(2-c_{1}-\Lambda a_{0}^{2}\right)+2 k\left(c_{1}^{2}-1\right)\right], \\
a_{l}= & \frac{1}{l^{2} c_{0}}\left[\frac{2}{3} \Lambda \sum_{j=0}^{l-1} \sum_{i=0}^{j} a_{i} a_{j-i} a_{l-1-j}-\frac{1}{3} a_{l-1}\right. \\
& \left.-\sum_{i=1}^{l} c_{i} a_{l-i}\left(l(l-i)+\frac{1}{6} i(i+1)\right)\right], \\
c_{l+1}= & \frac{3}{k(l+2)(l+1) l(l-1)} \\
& \times \sum_{i=0}^{l-1} a_{i} a_{l-i}(l-i)(l-1-3 i), \quad \text { for } \quad l \geq 2,
\end{aligned}
$$

with three free parameters $a_{0}, c_{0}, c_{1}$.

To identify the Schwarzschild-(anti-)de Sitter spacetime (3.5) in the form (3.4) with (4.1), first we evaluate the Bach tensor 2.8) on the horizon, yielding $\mathcal{B}_{1}\left(r_{h}\right)=0$, $\mathcal{B}_{2}\left(r_{h}\right)=-\frac{3}{k} a_{0}^{2} b$, where $b \equiv \frac{1}{3}\left(c_{1}-2+\Lambda a_{0}^{2}\right)$. Interestingly, by setting $b=0$ (i.e. for $c_{1}=2-\Lambda a_{0}^{2}$ ), the Bach tensor vanishes everywhere. Employing the gauge freedom of 2.2 , we may also set

$$
a_{0}=-\frac{1}{r_{h}}, \quad c_{0}=r_{h}-\frac{\Lambda}{r_{h}} .
$$

The explicit solution (3.4, 4.1) for $b=0$ then becomes

$$
\Omega(r)=-\frac{1}{r}, \quad \mathcal{H}(r)=\frac{\Lambda}{3}-r^{2}-\left(\frac{\Lambda}{3}-r_{h}^{2}\right) \frac{r^{3}}{r_{h}^{3}},
$$

where the expansions (3.4) were summed-up as geometric series. This is exactly the Schwarzschild-(anti-)de Sitter black hole (3.5) since $\frac{\Lambda}{3}-r_{h}^{2}=2 m r_{h}^{3}$.

In the case $b \neq 0$, we may now separate the 'Bach contribution' in the coefficients (4.1) proportional to $b$ by introducing $\alpha_{i}, \gamma_{i}$. With the same gauge choice (4.2), we obtain a one-parameter extension of the Schwarzschild(A)dS spacetime in quadratic gravity,

$$
\begin{aligned}
\Omega(r)=-\frac{1}{r}-\frac{b}{r_{h}} \sum_{i=1}^{\infty} \alpha_{i}\left(\frac{r_{h}-r}{\rho r_{h}}\right)^{i}, \\
\mathcal{H}(r)=\left(r-r_{h}\right)\left[\frac{r^{2}}{r_{h}}-\frac{\Lambda}{3 r_{h}^{3}}\left(r^{2}+r r_{h}+r_{h}^{2}\right)\right. \\
\left.+3 b \rho r_{h} \sum_{i=1}^{\infty} \gamma_{i}\left(\frac{r-r_{h}}{\rho r_{h}}\right)^{i}\right],
\end{aligned}
$$

where

$$
\begin{aligned}
\rho & \equiv 1-\frac{\Lambda}{r_{h}^{2}}, \\
\alpha_{1} & \equiv 1, \quad \gamma_{1}=1, \quad \gamma_{2}=\frac{1}{3}\left[4-\frac{1}{r_{h}^{2}}\left(2 \Lambda+\frac{1}{2 k}\right)+3 b\right],
\end{aligned}
$$

and $\alpha_{l}, \gamma_{l+1}$ are (with $\left.\alpha_{0} \equiv 0\right)$ recursively given by 


$$
\begin{aligned}
& \alpha_{l}=\frac{1}{l^{2}}[-\frac{2 \Lambda}{3 r_{h}^{2}} \sum_{j=0}^{l-1} \sum_{i=0}^{j}\left[\alpha_{l-1-j} \rho^{j}+\left(\rho^{l-1-j}+b \alpha_{l-1-j}\right)\left(\alpha_{i} \rho^{j-i}+\alpha_{j-i}\left(\rho^{i}+b \alpha_{i}\right)\right)\right]-\frac{1}{3} \alpha_{l-2}(2+\rho) \rho(l-1)^{2} \\
&\left.+\alpha_{l-1}\left[\frac{1}{3}+(1+\rho)\left(l(l-1)+\frac{1}{3}\right)\right]-3 \sum_{i=1}^{l}(-1)^{i} \gamma_{i}\left(\rho^{l-i}+b \alpha_{l-i}\right)\left(l(l-i)+\frac{1}{6} i(i+1)\right)\right], \\
& \gamma_{l+1}=\frac{(-1)^{l}}{k r_{h}^{2}(l+2)(l+1) l(l-1)} \sum_{i=0}^{l-1}\left[\alpha_{i} \rho^{l-i}+\alpha_{l-i}\left(\rho^{i}+b \alpha_{i}\right)\right](l-i)(l-1-3 i), \quad \text { for } \quad l \geq 2 .
\end{aligned}
$$

All these solutions form a three-parameter family of spherically symmetric black holes (with static regions). In particular:

- The radius $r=r_{h}$ determines the Killing horizon since $\mathcal{H}\left(r_{h}\right)=0$, see 4.5, 2.5.

- The parameter $\Lambda=R / 4$ is the cosmological constant. It can be zero, recovering the results of [19].

- The Bach parameter $b$ determines the Bach tensor contribution. For $b=0$, this Schwa-Bach-(A)dS black hole (4.4), 4.5 reduces to 4.3.

In terms of these three physical parameters, the scalar invariants (2.6), (2.7) on the horizon are

$$
\begin{aligned}
C_{a b c d} C^{a b c d}\left(r_{h}\right) & =12\left((1+b) r_{h}^{2}-\frac{1}{3} \Lambda\right)^{2}, \\
B_{a b} B^{a b}\left(r_{h}\right) & =\frac{r_{h}^{4}}{4 k^{2}} b^{2} .
\end{aligned}
$$

In Fig. 1. convergence of the series in (4.4), 4.5) is examined using the d'Alembert ratio test for two different sets of parameters. It clearly indicates that, with $n$ growing, the ratio between two subsequent terms approaches a specific constant. The series thus asymptotically behave as geometric series. This enables us to estimate the radius of convergence.

Typical behaviour of the metric function $\mathcal{H}(r)$ outside the black-hole horizon is plotted in Fig. 2. There is a significant qualitative difference between $\Lambda<0$ and $\Lambda>0$. In both cases, the black-hole horizon separates static $\left(r>r_{h}\right)$ and non-static $\left(r<r_{h}\right)$ regions of the spacetime. However, for $\Lambda>0$ an outer boundary of this static region appears, which corresponds to the cosmological horizon given by the second root of $\mathcal{H}$ (as in the classic Schwarzschild-de Sitter black hole). This is also demonstrated in Fig. 3 by plotting the function $f(\bar{r})$ of the common metric 2.1.

\section{SPECIFIC TIDAL EFFECTS}

The two independent parts 2.8 of the Bach tensor $\mathcal{B}_{1}, \mathcal{B}_{2}$ can be observed via a specific relative motion of free test particles described by the equation of geodesic deviation 27. For an invariant description, we employ an orthonormal frame associated with initially static observer $(\dot{r}=\dot{\theta}=\dot{\phi}=0)$ with velocity $\boldsymbol{u}=\dot{u} \partial_{u} \equiv \boldsymbol{e}_{(0)}$, namely $\boldsymbol{e}_{(1)}=-\dot{u}\left(\partial_{u}+\mathcal{H} \partial_{r}\right)$, $\boldsymbol{e}_{(2)}=\Omega^{-1} \partial_{\theta}$, and $\boldsymbol{e}_{(3)}=(\Omega \sin \theta)^{-1} \partial_{\phi}$. Projection of the equation of geodesic deviation onto this frame gives

$$
\begin{aligned}
& \ddot{Z}^{(1)}=\frac{\Lambda}{3} Z^{(1)}+\frac{1}{6} \frac{\mathcal{H}^{\prime \prime}+2}{\Omega^{2}} Z^{(1)}-\frac{k}{3} \frac{\mathcal{B}_{1}+\mathcal{B}_{2}}{\Omega^{4}} Z^{(1)}, \\
& \ddot{Z}^{(i)}=\frac{\Lambda}{3} Z^{(i)}-\frac{1}{12} \frac{\mathcal{H}^{\prime \prime}+2}{\Omega^{2}} Z^{(i)}-\frac{k}{6} \frac{\mathcal{B}_{1}}{\Omega^{4}} Z^{(i)}
\end{aligned}
$$

where $i=2,3, Z^{(a)} \equiv e^{(a)}{ }_{\mu} Z^{\mu}$ denotes relative position of two particles, and $\ddot{Z}^{(a)} \equiv e^{(a)}{ }_{\mu} \frac{\mathrm{D}^{2} Z^{\mu}}{\mathrm{d} \tau^{2}}$ their mutual acceleration. In (5.1), 5.2), we easily identify classic parts corresponding to the isotropic influence of the cosmological constant $\Lambda$ and the Newtonian tidal effect caused by the Weyl tensor proportional to the square root of 2.6. Moreover, the theory satisfying (1.3) admits two additional effects encoded in the non-trivial Bach tensor components $\mathcal{B}_{1}, \mathcal{B}_{2}$. The first of them affects particles in the transverse directions $\partial_{\theta}, \partial_{\phi}$, see 5.2 , while the second one induces their radial acceleration along $\partial_{\bar{r}}$ via 5.1. Since $\mathcal{B}_{1}\left(r_{h}\right)=0$, on any horizon there is only the radial effect caused by $\mathcal{B}_{2}\left(r_{h}\right)$.

\section{THERMODYNAMIC QUANTITIES: HORIZON AREA, TEMPERATURE, ENTROPY}

Let us also determine main thermodynamic properties of this explicit family of spherically symmetric SchwaBach-(A)dS black holes. The horizon is generated by the (rescaled) null Killing vector $\ell \equiv \sigma \partial_{u}=\sigma \partial_{t}$ and thus is located at $r=r_{h}$ where $\mathcal{H}=0$, cf. (2.5), 4.5). Its area is, using (2.2), (4.4),

$$
\mathcal{A}=4 \pi r_{h}^{-2}=4 \pi \bar{r}_{h}^{2},
$$

while its surface gravity $\left(\kappa^{2} \equiv-\frac{1}{2} \ell_{\mu ; \nu} \ell^{\mu ; \nu}\right)$ reads

$$
\kappa / \sigma=-\frac{1}{2} \mathcal{H}^{\prime}\left(r_{h}\right)=-\frac{1}{2} \rho r_{h}=\frac{1}{2} \bar{r}_{h}^{-1}\left(1-\Lambda \bar{r}_{h}^{2}\right) .
$$

It is the same expression as in the Schwarzschild- $(A) d S$ case, independent of the Bach parameter $b$. The blackhole horizon temperature $T=\frac{1}{2 \pi} \kappa$ is thus

$$
T / \sigma=-\frac{1}{4 \pi} \rho r_{h}=\frac{1}{4 \pi} \bar{r}_{h}^{-1}\left(1-\Lambda \bar{r}_{h}^{2}\right) .
$$




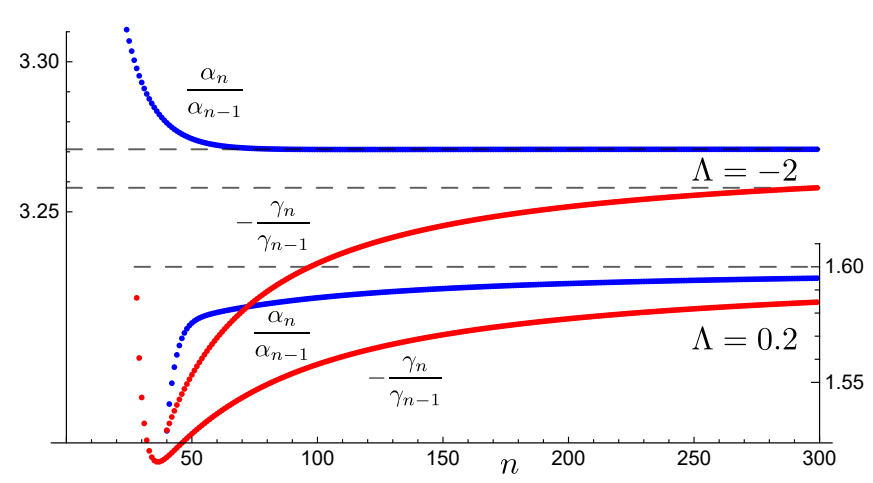

FIG. 1. The convergence radius can be estimated from the ratio convergence test for solutions 4.4, 4.5, here given by $r_{h}=-1, k=0.5$ with $b=0.3, \Lambda=0.2$ (bottom) and $b=0.2, \Lambda=-2$ (top).

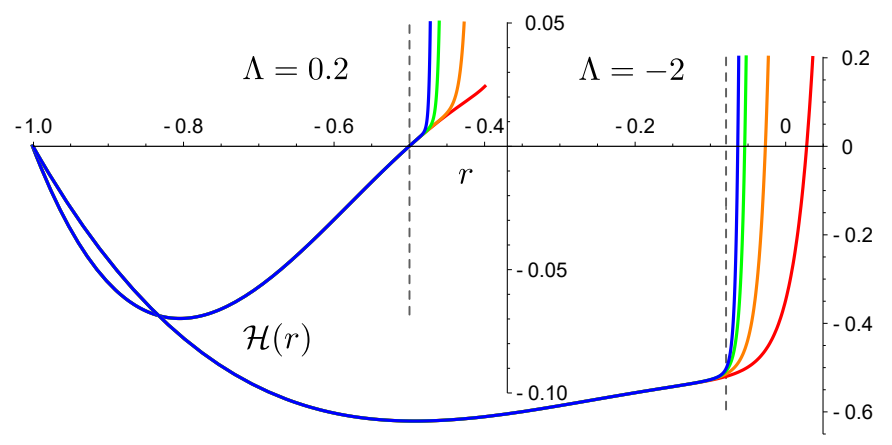

FIG. 2. The function $\mathcal{H}(r)$ given by 4.5 for two values of the cosmological constant $\Lambda$ (with the same parameters as in Fig. 1). Both plots start on the black-hole horizon $r_{h}=-1$ and are reliable up to the vertical dashed lines indicating the radii of the convergence. For $\Lambda>0$ the function $\mathcal{H}(r)$ seems to have another root corresponding to the cosmological horizon, while for $\Lambda<0$ it remains non-vanishing. First 50 (red), 100 (orange), 200 (green), 300 (blue) terms in the expansions are used. The results fully agree with the numerical solutions up to the dashed lines, where such simulations also fail.

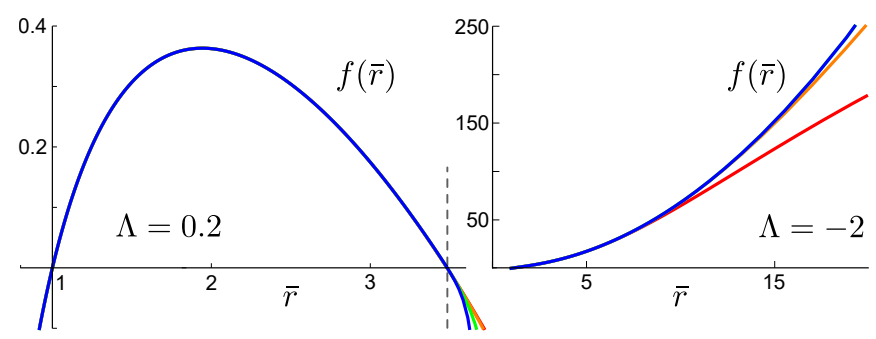

FIG. 3. The function $f(\bar{r})$ of standard line element 2.1) corresponding to the solution (4.4), 4.5) via 2.4) (with the same parameters as in Figs. 1, 2). The $\Lambda>0$ case (left) indicates the presence of the cosmological horizon at the boundary of the convergence interval (the dashed line). For $\Lambda<0$ (right), the series converge in the whole plotted range, indicating a static region everywhere above the black-hole horizon.
This is zero for $\bar{r}_{h}=1 / \sqrt{\Lambda}$ corresponding to the case of extreme Schwarzschild-de Sitter black hole for which the black-hole and cosmological horizons coincide at $\bar{r}_{h}=\bar{r}_{c}$.

However, in higher-derivative theories, we must apply the generalized definition of entropy $S=(2 \pi / \kappa) \oint \mathbf{Q}$, see [28, where the Noether charge 2-form on the horizon is

$$
\begin{aligned}
\mathbf{Q}=-\frac{\Omega^{2} \mathcal{H}^{\prime}}{16 \pi}\left[\gamma+\frac{4}{3} \Lambda(\alpha+6 \beta)+\right. & \left.\frac{4}{3} k \alpha \frac{\mathcal{B}_{1}+\mathcal{B}_{2}}{\Omega^{4}}\right]\left.\right|_{r=r_{h}} \\
& \times \sin \theta \mathrm{d} \theta \wedge \mathrm{d} \phi .
\end{aligned}
$$

Evaluating the integral, using (6.1), 6.2, 4.9) and $r_{h}=-1 / \bar{r}_{h}$, we get

$$
S=\frac{1}{4} \mathcal{A}\left[\gamma+\frac{4}{3} \Lambda(\alpha+6 \beta)-4 \alpha \frac{b}{\bar{r}_{h}^{2}}\right]
$$

For the Schwarzschild black hole $(b=0, \Lambda=0)$ or in the Einstein theory $(\alpha=0, \beta=0)$, this reduces to the standard expression $S=\frac{1}{4 G} \mathcal{A}$. For $\Lambda=0$, the results of 14, 19, are recovered. For the Schwarzschild-(A)dS black hole $(b=0)$ in Einstein-Weyl gravity $(\beta=0)$, we obtain $S=\frac{1}{4 G} \mathcal{A}\left(1+\frac{4}{3} k \Lambda\right)$, which agrees with the results of [29]. In critical gravity, defined by $\beta=0, \alpha=k \gamma$, $\Lambda=-\frac{3}{4 k}<0$, the entropy is zero. Our formula 6.5 for entropy generalizes all these expressions to the case of Schwarzschild-Bach-(anti-)de Sitter black holes when the Bach tensor is non-vanishing, parameterized by $b \neq 0$. In this case, the entropy is non-zero even in critical gravity. For smaller black holes, the deviations from $S=\frac{1}{4} \mathcal{A}\left[\gamma+\frac{4}{3} \Lambda(\alpha+6 \beta)\right]$ are larger.

By replacing the root $r_{h}$ by $r_{c}$ in (4.4), (4.5), the solution is expanded around the cosmological horizon. Its temperature and entropy are thus given by $(6.3)$ and 6.5), respectively, in which $\bar{r}_{h}$ is simply replaced by $\bar{r}_{c}$.

\section{ACKNOWLEDGEMENTS}

This work has been supported by the Czech Science Foundation Grant No. GAČR 17-01625S and the CzechAustrian MOBILITY grant 8J18AT02 (JP, RŠ), and the Research Plan RVO: 67985840 (VP, AP). We thank $\mathrm{H}$. Maeda for reading the manuscript. We are also grateful to anonymous referees for their very useful comments, observations and suggestions. 
[1] Einstein A 1916 Die Grundlage der allgemeinen Relativitätstheorie, Ann. der Physik 49769

[2] Schwarzschild K 1916 Über das Gravitationsfeld eines Massenpunktes nach der Einsteinschen Theorie, Sitz. Preuss. Akad. Wiss. Berlin 7189

[3] Kerr R P 1963 Gravitational field of a spinning mass as an example of algebraically special metrics, Phys. Rev. Lett. 11237

[4] Einstein A 1917 Kosmologische Betrachtungen zur allgemeinen Relativitätstheorie, Sitz. Preuss. Akad. Wiss. Berlin 142

[5] de Sitter W 1917 Over de relativiteit der traagheid: Beschouingen naar aanleiding van Einstein's hypothese, Koninklijke Akademie van Wetenschappen te Amsterdam 25 1268; 1918 Proc. Akad. Amsterdam 191217

[6] Schrödinger E 1956 Expanding universes (Cambridge: Cambridge University Press)

[7] Sotiriou T P and Faraoni V $2010 f(R)$ theories of gravity, Rev. Mod. Phys. 82451

[8] De Felice A and Tsujikawa S $2010 f(R)$ Theories, Living Rev. Relativity $\mathbf{1 3} 3$

[9] Capozziello S and De Laurentis M 2011 Extended Theories of Gravity, Physics Reports 509167

[10] Clifton T et al. 2012 Modified gravity and cosmology, Physics Reports $\mathbf{5 1 3} 1$

[11] Tangherlini F R 1963 Schwarzschild Field in $n$ Dimensions and the Dimensionality of Space Problem, Nuovo Cim. 77636

[12] Myers R C and Perry M J 1986 Black Holes in Higher Dimensional SpaceTimes, Annals Phys. 172304

[13] Boulware D G and Deser S 1985 String-Generated Gravity Models, Phys. Rev. Lett. 552656

[14] Lü H, Perkins A, Pope C N and Stelle K S 2015 Black holes in higher derivative gravity, Phys. Rev. Lett. 114 171601

[15] Stelle K S 1978 Classical gravity with higher derivatives, Gen. Relativ. Gravit. 9353
[16] Weyl H 1919 Eine neue Erweiterung der Relativitätstheorie, Ann. der Physik 59101

[17] Bach R 1921 Zur Weylschen Relativitätstheorie und der Weylschen Erweiterung des Krümmungstensorbegriffs, Math. Zeitschrift 9110

[18] Kottler F 1918 Über die physikalischen Grundlagen der Einsteinschen Gravitationstheorie, Ann. Physik 56401

[19] Podolský J, Švarc R, Pravda V and Pravdová A 2018 Explicit black hole solutions in higher-derivative gravity, Phys. Rev. D 98 021502(R)

[20] Codello A and Percacci R 2006 Fixed Points of HigherDerivative Gravity, Phys. Rev. Lett. 97221301

[21] Pravda V, Pravdová A, Podolský J and Švarc R 2017 Exact solutions to quadratic gravity, Phys. Rev. D 95 084025

[22] Podolský J, Švarc R, Pravda V and Pravdová A 2018 (in preparation)

[23] Mannheim P D and Kazanas D 1989 Exact vacuum solution to conformal Weyl gravity and galactic rotation curves, Astrophysical Journal 342635

[24] Stephani H, Kramer D, MacCallum M, Hoenselaers C, and Herlt E 2003 Exact Solutions of Einstein's Field Equations (Cambridge: Cambridge University Press).

[25] Griffiths J and Podolský J 2009 Exact Space-Times in Einstein's General Relativity (Cambridge: Cambridge University Press)

[26] Pravda V, Pravdová A, Podolský J and Švarc R 2018 (in preparation)

[27] Podolský J and Švarc R 2012 Interpreting spacetimes of any dimension using geodesic deviation, Phys. Rev. D 85 044057

[28] Wald R M 1993 Black hole entropy is the Noether charge, Phys. Rev. D 48 R3427(R)

[29] Lü H and Pope C N 2011 Critical gravity in four dimensions, Phys. Rev. Lett. 106181302 